\title{
Successful treatment of a mycotic aortic pseudoaneurysm in a patient with type 2 diabetes mellitus while treating primary aldosteronism with spironolactone
}

\author{
Yuzuru Ito ${ }^{1,2}$, Koichiro Yoshimura ${ }^{1}$, Yoko Matsuzawa ${ }^{1}$, Jun Saito ${ }^{1}$, Hiroko Ito ${ }^{1}$, Hiroshi Furukawa ${ }^{3}$, \\ Kazuhiro Okura ${ }^{3}$, Mutsumu Fukata ${ }^{3}$, Toshio Konishi ${ }^{3}$, and Tetsuo Nishikawa ${ }^{1}$ \\ ${ }^{1}$ Division of Endocrinology \& Metabolism. Department of Medicine, Yokohama Rosai Hospital, Yokohama, Japan \\ ${ }^{2}$ Department of Endocrinology and Metabolism. Yokohama City University Graduate School of Medicine, Yokohama, Japan \\ ${ }^{3}$ Department of Cardiovascular Surgery, Yokohama Rosai Hospital, Yokohama, Japan
}

\begin{abstract}
We describe a diabetic patient successfully treated for an acute mycotic aortic arch pseudoaneurysm with primary aldosteronism. The patient first complained of severe pain in the left upper extremity and left back with high $\mathrm{C}$ reactive protein (CRP) and high-grade fever. It was suspected that acute aortic dissection had developed in association with mycotic pseudoaneurysm of the aortic arch because of chest X-ray findings of enlargement of the aortic arch. Computed tomography (CT) of the aortic arch revealed an aortic aneurysm protruding in the superior direction. Staphylococcus aureus was detected in blood culture, suggesting a mycotic aortic aneurysm, and artificial blood vessel replacement of the aortic arch was performed. Intraoperative findings suggested aortic pseudoaneurysm, which consisted of mediastinal rupture of the aorta at the distal arch. Our patient had a 2-year history of type 2 diabetes mellitus and poor blood sugar control, even with twice-daily injection of insulin. Blood pressure was not always well controlled because of primary aldosteronism. Thus, it was speculated that hyperaldosteronism, as well as diabetes-associated atherosclerosis, had persisted for a long time. No reports have described mycotic pseudoaneurysm in the aortic arch in a diabetic patient associated with primary aldosteronism. It is necessary to note that serious vascular complications are possible if aldosteronism is left untreated or is treated insufficiently as essential hypertension.
\end{abstract}

J Atheroscler Thromb, 2010; 17:771-775.

Key words; Mycotic aortic pseudoaneurysm, Type 2 diabetes mellitus, Primary aldosteronism

\section{Introduction}

Osler first coined the term "mycotic aneurysm" in 1885 to describe a mushroom-shaped aneurysm in a patient with endocarditis ${ }^{1)}$. Mycotic pseudoaneurysm of the ascending aorta is a rare complication after cardiac surgery and is caused by the growth of bacteria or fungi in the vessel wall. Such aneurysms are more likely to occur in immunosuppressed patients after heart or heart-lung transplantation than after coro-

Address for correspondence: Tetsuo Nishikawa, Department of Medicine, Yokohama Rosai Hospital, 3211 Kozukue-cho, Kohoku-ku,Yokohama City 222-0036, Japan

E-mail: tatsuon@yokohamah.rofuku.go.jp

Received: October 20, 2009

Accepted for publication: December 22, 2009 nary artery bypass grafting $(\mathrm{CABG})^{2)}$. Whereas Candida species and Gram-negative bacteria are more common after heart or heart-lung transplantation, Staphylococcus aureus is the organism most frequently found after $\mathrm{CABG}^{2)}$. Pseudomonas aeruginosa has rarely been reported in an ascending aorta mycotic pseudoaneurysm $^{3)}$. We experienced a diabetic patient successfully treated for an acute mycotic aortic arch pseudoaneurysm with aldosteronism. Artificial blood vessel replacement of the aortic arch was performed 4 days after admission. Intraoperative findings suggested an aortic pseudoaneurysm, which consisted of mediastinal rupture of the aorta at the distal arch. We here report an interesting and very rare case of mycotic pseudoaneurysm in the aortic arch in a diabetic patient associated with primary aldosteronism, who initially 
Table 1. Endocrine Data

\begin{tabular}{|c|c|}
\hline Plasma ACTH (pg/mL) & $25.0(<60)$ \\
\hline Plasma cortisol $(\mu \mathrm{g} / \mathrm{dL})$ & $5.5(5.6-21.3)$ \\
\hline PAC (ng/dL) & $5.7(2.0-13.0)$ \\
\hline PRA (ng/mL/hr) & $<0.1(0.2-2.7)$ \\
\hline Plasma adrenalin (ng/mL) & $0.04(<0.10)$ \\
\hline Plasma noradrenalin $(\mathrm{ng} / \mathrm{mL})$ & $0.14(0.05-0.4)$ \\
\hline Plasma dopamine (ng/mL) & $0.02(<0.02)$ \\
\hline Captopril (50 mg) test & $90 \mathrm{~min}$ \\
\hline PAC (ng/dL) & 6.6 \\
\hline PRA (ng/mL/hr) & 0.2 \\
\hline Furosemide (40 mg) plus upright test & $120 \mathrm{~min}$ \\
\hline $\mathrm{PAC}(\mathrm{ng} / \mathrm{dL})$ & 10.8 \\
\hline PRA (ng/mL/hr) & $<0.1$ \\
\hline
\end{tabular}

Adrenal venous sampling (before/30 min after ACTH (0.25 mg) stimulation)

\begin{tabular}{lccc} 
& right adrenal vein & left adrenal vein & inferior vena cava \\
\cline { 2 - 4 } PAC $(\mathrm{ng} / \mathrm{dL})$ & $387 / 1,843$ & $107 / 748$ & $5 / 16$ \\
corticol $(\mu \mathrm{g} / \mathrm{dL})$ & $124 / 770$ & $138 / 418$ & $6 / 15$ \\
\hline
\end{tabular}

PAC, plasma aldosterone concentration; PRA, plasma renin activity.

Captopril test and furosemide plus upright test were performed as reported previously ${ }^{5,6)}$.

Parentheses show normal range of each hormone.

showed nonspecific left upper limb pain.

\section{Case Presentation}

A female patient aged 69 years old complained of fever, left upper limb pain and a high-grade fever of around $38.5^{\circ} \mathrm{C}$ when she visited our outpatient clinic in 2004. Her past history showed hypertension and uterine myoma at 46 years of age, acute pancreatitis at 65 , primary aldosteronism at 66 , and diabetes mellitus at 67 . She had smoked 80 cigarettes/day between 25 and 65 years old. The patient had consulted the outpatient clinic of our hospital to treat hypertension and diabetes mellitus two years previously.

Primary aldosteronism was diagnosed at 66 years of age. Serum potassium was $3.8 \mathrm{mEq} / \mathrm{L}$. Plasma renin activity (PRA) was undetectable and the aldosterone level was elevated so that the aldosterone-renin ratio (ARR) was markedly high. Diagnosis of primary aldosteronism was evident at this stage. Left ventricular hypertrophy on ECG, cardiomegaly (CTR 58\%) on chest X-ray, and hypertensive retinopathy (KeithWagener II) on funduscopic examination were considered complications of long-standing hypertension. PRA remained suppressed $(0.4 \mathrm{ng} / \mathrm{mL} /$ hour $)$ after stimulation with furosemide $(40 \mathrm{mg})$ plus an upright posture for 2 hours (Table 1), and was still low (0.2

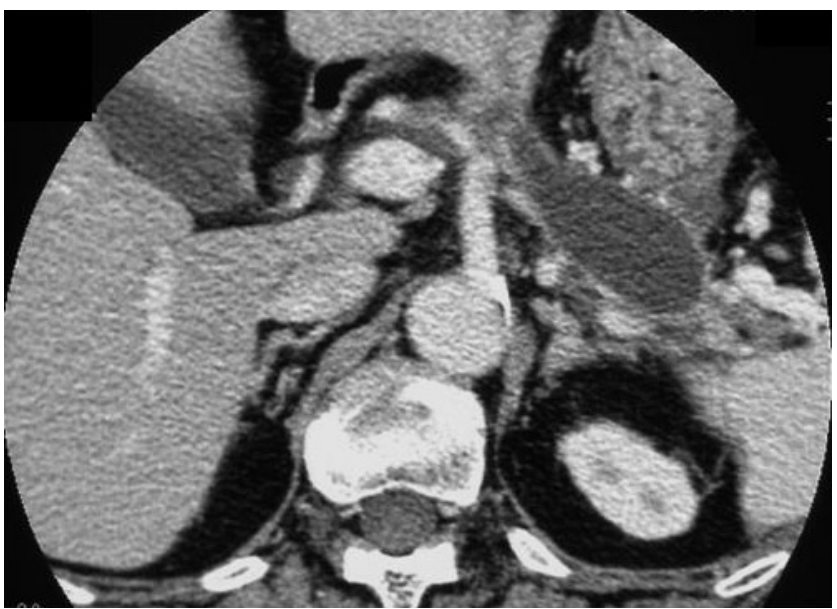

Fig. 1. Abdominal CT showed almost normal adrenal glands.

$\mathrm{ng} / \mathrm{mL} /$ hour) after administration of $50 \mathrm{mg}$ captopril. Computed tomography (CT) showed no abnormal lesions in either adrenal gland (Fig. 1). To definitely diagnose primary aldosteronism, adrenal venous sampling before and $30 \mathrm{~min}$ after adrenocorticotrophic hormone (ACTH) stimulation was performed according to the method reported previously ${ }^{4)}$. Basal aldosterone levels in right and left adrenal veins were 387 and $107 \mathrm{ng} / \mathrm{dL}$, respectively, which rose to 1,843 and 748 


\section{June, 2001}

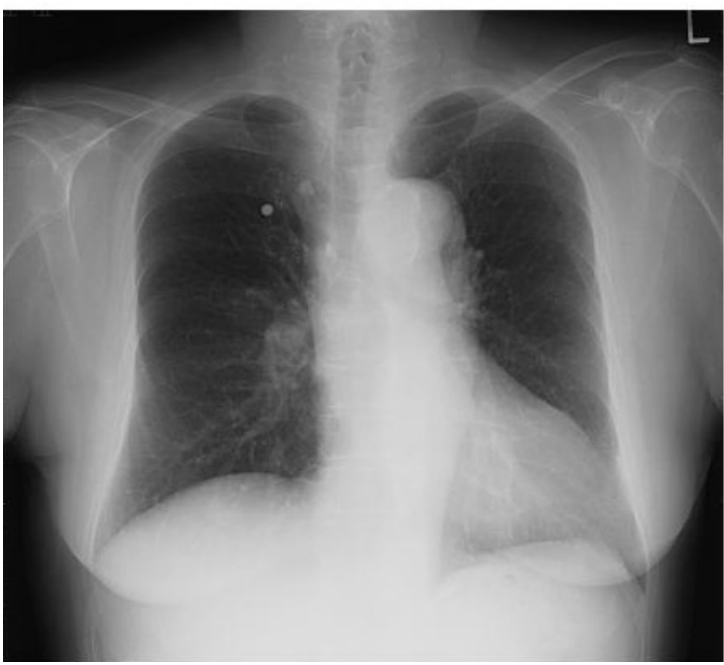

December, 2003

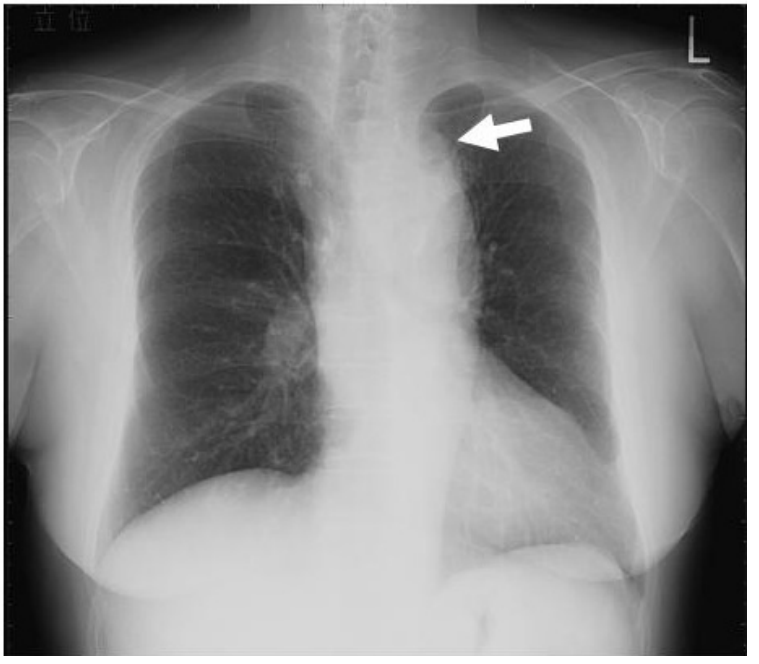

Fig. 2. Chest X-ray from June, 2001 (left) and December, 2003 (right). Arrow shows aortic pseudoaneurysm.

ng/dl, respectively, after stimulation with ACTH. These data suggested aldosterone hypersecretion from the right adrenal gland. We recommended unilateral resection of the right adrenal gland, but she refused, so spironolactone $(25 \mathrm{mg} /$ day $)$ was administered to treat hyperaldosteronemia. This caused an increase in PRA (3.9 ng/mL/hour) and plasma aldosterone concentration (PAC) $(16.7 \mathrm{ng} / \mathrm{dL}) ; 20 \mathrm{mg}$ nifedipine CR and $25 \mathrm{mg}$ spironolactone had been administered, and her blood pressure had been controlled to 110-130/70$80 \mathrm{mmHg}$. As diabetes treatment, the HbAlc value had been controlled to approximately $7 \%$ by Novolin $30 \mathrm{R} 8$ units in the morning and 6 units in the evening.

In early December 2004, at 69 years of age, cold symptoms developed. On December 4, pain in the left upper half of the body, involving the shoulder to fingers, occurred. Administration of loxoprofen sodium did not improve the symptoms and, in mid-December, she consulted our hospital. Her white blood cell count (WBC), C reactive protein (CRP), and blood sugar were $24,500 / \mu \mathrm{L}, 47.5 \mathrm{mg} / \mathrm{dL}$ and $474 \mathrm{mg} / \mathrm{dL}$, respectively, suggesting severe infection and hyperglycemia. She was therefore admitted as an emergency. On admission, blood pressure, pulse rate and body temperature were $118 / 68 \mathrm{mmHg}, 94 / \mathrm{min}$ and $36.4^{\circ} \mathrm{C}$, respectively, after oral administration of loxoprofen sodium. Physical examination was unremarkable except for tenderness of the left scapula. The urinary ketone body level was $2+$ without acidosis in arterial blood. ECG showed incomplete right bundle branch block and left ventricular hypertrophy. On chest X-ray, the cardio-thoracic rate was 58\%. There was a marked protrusion of the first left arch (Fig. 2).

\section{Clinical course after admission}

After admission, she had a $38^{\circ} \mathrm{C}$ or higher fever and a very high level of inflammatory markers, so the source of infection was investigated. Contrast-enhanced thoracic CT revealed an aortic arch aneurysm $5.3 \mathrm{~cm}$ in diameter, protruding in the superior direction, and calcification in the aorta (Fig. 3A, B). Staphylococcus aureus was detected in blood culture, suggesting an infectious aortic aneurysm. Pain in the left scapular lesion persisted. With combination antibiotics therapy, artificial blood vessel replacement of the aortic arch was performed 4 days after admission. Intraoperative findings suggested that the aortic pseudoaneurysm had developed because of massive bleeding from mediastinal rupture of the aorta at the distal arch. Aneurysmal pus culture identified Staphylococcus aureus. The operation accompanied immediate improvement in the WBC and CRP values. The subsequent course was uneventful except for mild aspiration pneumonia, and she was discharged 65 days after admission without recurrence until 2006 (Fig.4).

\section{Discussion}

We report here an interesting and very rare case of mycotic pseudoaneurysm in the aortic arch in a di- 

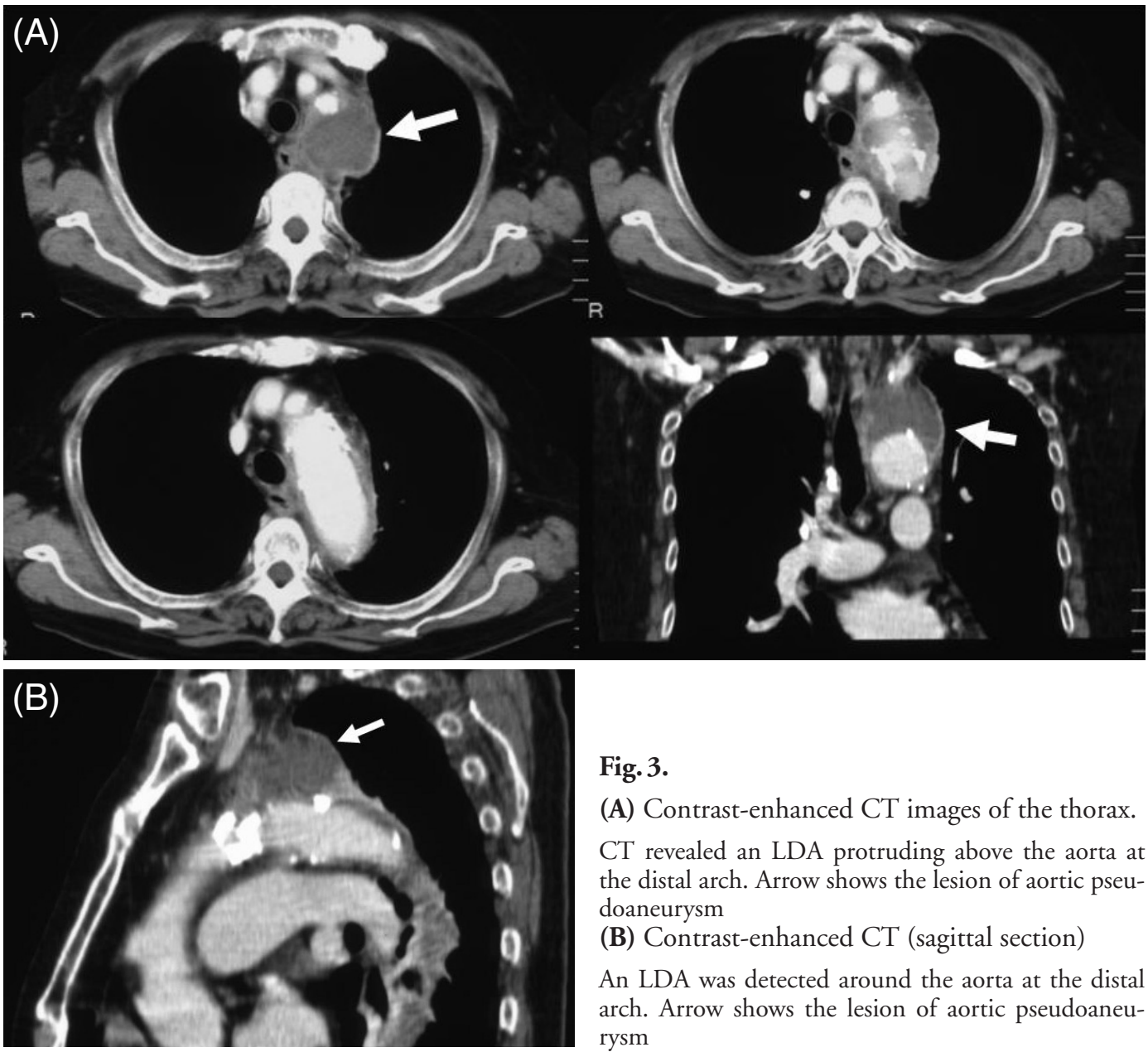

Fig. 3.

(A) Contrast-enhanced CT images of the thorax.

CT revealed an LDA protruding above the aorta at the distal arch. Arrow shows the lesion of aortic pseudoaneurysm

(B) Contrast-enhanced CT (sagittal section)

An LDA was detected around the aorta at the distal arch. Arrow shows the lesion of aortic pseudoaneurysm

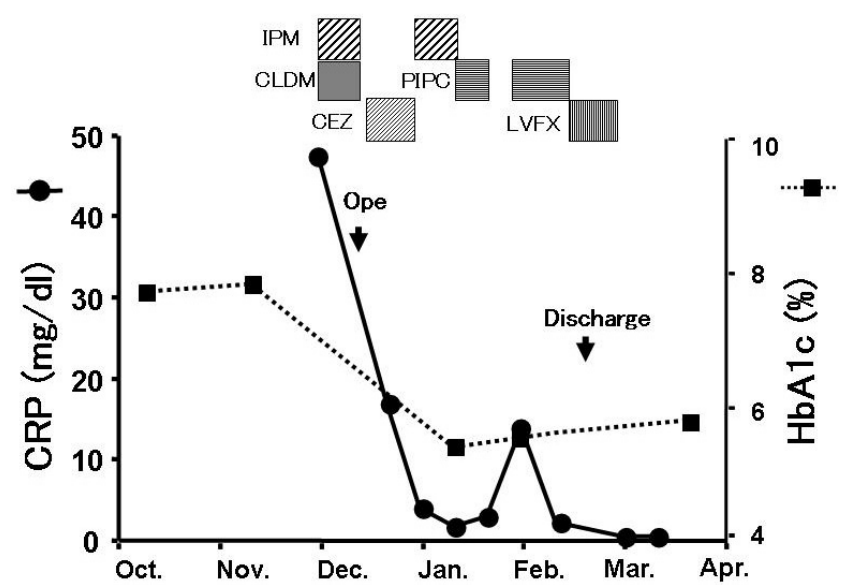

Fig. 4. Clinical course.

Changes in CRP and HbAlc levels. Upper section shows the administered antibiotics. Patient was admitted in mid-December. Operation (Ope) was performed 4 days after admission, and the patient was discharged 65 days after admission.

O. CRP (mg/dl); $\mathbf{\square}$, HbAlc (\%). IPM, imipenem; CLDM, clindamycin; PIPC, piperacillin; CEZ, cefazolin; LVFX, levofloxacin. abetic patient with primary aldosteronism. Infection control with antibiotics should be started immediately after diagnosis, and surgical treatment should be performed as soon as possible. In the present case, pain in the left upper limb had persisted for 2 weeks before admission, suggesting rapid expansion of the aneurysm.

It has been reported that the source and route of infection in mycotic aortic pseudoaneurysms may be 1) cardiovascular infection-related embolism (infectious endocarditis, bacteremia), 2) direct transmission from the peripheral infectious focus (pneumonia, abscess, intraoperative infection), and 3) intravascular operation- or angiography-related aortic infection. In the present patient, the infection focus was unclear; however, she had complained of tooth pain a few weeks before admission, suggesting gingivitis. In addition, she had a history of fracture of the fifth left finger a few months before admission. These events might have resulted in bacteremia leading to the mycotic pseudoaneurysm. Calcification around the aneurysm 
from the findings of CT may induce bacterial adhesion via damage to the intimal function. Primary aldosteronism is a curable form of secondary hypertension, and the incidence of primary aldosteronism among hypertensives is higher than previously reported ${ }^{5)}$. It has been reported that aldosterone plays a crucial role in the development of damaged organs following heart failure, fibrotic changes in blood vessels, and renal fail$u^{7}{ }^{7)}$, and that primary hyperaldosteronism is a potential independent risk factor for aortic dissection ${ }^{8)}$. There are several reports of patients with primary aldosteronism, which caused dissecting aortic aneurysm $^{9,10)}$. The present patient had been suffering from hypertension for 20 years before the definite diagnosis of primary aldosteronism, and blood pressure was not always well controlled. Thus, it can be considered that hypertension and hyperaldosteronemia had persisted for a long time, which might have been sufficient to induce various dysfunctions of the vascular endothelium and smooth muscle cells.

Moreover, she had smoked for a long time, which is also a risk factor for vascular damage. Even after treatment with spironolactone, persistent hyperaldosteronemia may partly account for her aortic pseudoaneurysm. It is necessary to note that serious vascular complications are possible if aldosteronism is left untreated or is treated insufficiently as essential hypertension. Thus, it is very important to attenuate hyperaldosteronemia in patients with primary aldosteronism to prevent various aldosterone-induced complications. It was unlikely to have caused such an aneurysm if surgery had been performed two years previously.

In conclusion, we report here the first case of mycotic aortic pseudoaneurysm that was successfully cured in a patient with type 2 diabetes associated with primary aldosteronism. In our patient, the development of aortic pseudoaneurysm was feasible with long-standing hypertension due to primary aldosteronism. We should always consider mycotic pseudoaneurysm in the aortic arch in a diabetic patient associated with primary aldosteronism, even after treating with an aldosterone blocker, such as spironolactone.

\section{Acknowledgements}

The present study was supported by a Grant-inAid for Scientific Research "Adrenal Disorders" from the Ministry of Public Health and Labor, Japan.

\section{References}

1) Yasuda K, Sasaki S: Mycotic aneurysm and mycotic aortitis. Ryoikibetsu Shokogun Shirizu, 1996; 14: 325-328 (in Japanese)

2) Schmitt TM, Finck SJ, Brumble LM, Lane GE: Pseudomonas aeruginosa pseudoaneurysm of the ascending aorta after coronary artery bypass graft surgery. Tex Heart Inst J, 2003; 30: 137-139

3) Vrochides D, Feng WC, Singh AK: Mycotic ascending aortic pseudoaneurysm secondary to pseudomonas mediastinitis at the aortic cannulation site. Tex Heart Inst J, 2003; 30: 322-324

4) Nishikawa T, Saito J, Soyama A, Ito H, Omura M: Occurrence and diagnosis of primary aldosteronism and its therapy. Nippon Naika Gakkai Zasshi, 2003; 92: 208-212 (in Japanese)

5) Omura M, Saito J, Yamaguchi K, Kakuta Y, Nishikawa T: Prospective study on the prevalence of secondary hypertension among hypertensive patients visiting a general outpatient clinic in Japan. Hypertens Res, 2004; 27: 193-202

6) Naomi S, Iwaoka T, Umeda T, Inoue J, Hamasaki S, Miura F, Fujii Y, Sato T: Clinical evaluation of the captopril screening test for primary aldosteronism. Jpn Heart J, 1985; 26: 549-556 (in Japanese)

7) Takeda R, Matsubara T, Miyamori I, Hatakeyama H, Morise T: Vascular complications in patients with aldosterone producing adenoma in Japan: comparative study with essential hypertension. The Research Committee of Disorders of Adrenal Hormones in Japan. J Endocrinol Invest, 1995; 18: 370-373

8) Ahmed SH, Husain NM, Khawaja SN, Massey CV, Pettyjohn FS: Is primary hyperaldosteronism a risk factor for aortic dissection? Cardiology, 2007; 108: 48-50

9) Safi AM, Kwan T, Afflu E, Alam M, Anderson JE, Clark LT: Coronary artery aneurysms, aortic dissection, and hypertension secondary to primary aldosteronism: a rare triad. Angiology, 1999; 50: 503-508

10) Shimizu A, Aoi W, Akahoshi M, Utsunomiya T, Doi $Y$, Suzuki S, Kuramochi M, Hashiba K: Elevation of plasma renin activity during pregnancy and rupture of a dissecting aortic aneurysm in a patient with primary aldosteronism. Jpn Heart J, 1983; 24: 995-1006 (in Japanese) 